Research Article

\title{
Development of an Immune-Related Risk Signature in Patients with Bladder Urothelial Carcinoma
}

\author{
Yaofei Jiang $\mathbb{D}^{1},{ }^{1,2}$ Yibing Wang $\mathbb{D},{ }^{3}$ Cong Li $\mathbb{D}^{1},{ }^{1}$ Zhenhong Zou $\mathbb{D}^{1},{ }^{1}$ and Bo Liang $\mathbb{D}^{1}$ \\ ${ }^{1}$ Department of General Surgery, Second Affiliated Hospital of Nanchang University, 1 Minde Road, Nanchang, \\ 330006 Jiangxi, China \\ ${ }^{2}$ Department of Radiation Oncology and Medical Oncology, Zhongnan Hospital, Wuhan University, Wuhan, China \\ ${ }^{3}$ Department of Emergency, Second Affiliated Hospital of Nanchang University, 1 Minde Road, Nanchang, 330006 Jiangxi, China
}

Correspondence should be addressed to Zhenhong Zou; zhenhongzou@163.com and Bo Liang; 2656304557@qq.com

Received 28 May 2020; Revised 20 July 2020; Accepted 4 August 2020; Published 20 August 2020

Academic Editor: Joshua R. Mauney

Copyright (c) 2020 Yaofei Jiang et al. This is an open access article distributed under the Creative Commons Attribution License, which permits unrestricted use, distribution, and reproduction in any medium, provided the original work is properly cited.

With recent advances in immunooncology and tumor microenvironment, the treatment landscape of bladder urothelial carcinoma has been changing dramatically. We aim to construct an immune gene-related signature which can predict BLCA patients' overall survival. Transcriptomic data of BLCA patients was downloaded from The Cancer Genome Atlas database, and immune-related genes were downloaded from the Immunology Database and Analysis Portal database. Prognostic immune-related genes were identified. We then constructed and validated an immune gene-related signature. Tumor-related transcription factors were downloaded from the Cistrome database, and a network between them and prognostic immune-related genes was generated. Cox's proportional hazards model and the Kaplan-Meier survival analysis were performed to assess our signature's prognostic ability. Relationship between the signature and patients' clinicopathologic features was then explored to validate its clinical value. We further downloaded concentration of six types of immune cells from the Tumor Immune Estimation Resource database to explore immune-related potential mechanisms of the signature.

\section{Introduction}

Bladder cancer is the most common cancer in the urinary tract and the ninth most common cancer worldwide, with 549393 new cases and 199922 deaths in 2018 [1,2]. There are 80470 new cases and 17670 deaths expected in the USA in 2019 according to the latest epidemiological data on bladder cancer [3]. Bladder urothelial carcinoma (BLCA) is the most common histopathological type of bladder cancer $[3,4]$. About a quarter of BLCA patients are muscle invasive bladder cancer (MIBC) and the rest are nonmuscle invasive bladder cancer (NMIBC) [5-7]. Surgery is one of the main treatments for BLCA. However, more than $30 \%$ of BLCA patients will experience tumor recurrence after surgery, which eventually bring bladder cancer $13^{\text {th }}$ most common cause of cancer death $[2,8]$. With high recurrence and metastasis feature, BLCA patients suffer from great mental and economic pressure, as well as severe physical pain. Therefore, further exploration of BLCA patients' diagnosis and treatment is urgently necessary.

There is an upsurge of interest in tumor immune microenvironment (TIM) in recent years. With either immune suppressor or immune promoter ability, a variety of immune cells make up TIM, which can limit T cells' accumulation at where cancer cells locate [9]. Interestingly, immunotherapy has been playing an important role in the treatment of BLCA for more than 40 years and intravesical Bacillus CalmetteGuerin (BCG) remains to be the most efficacious intravesical medicament for NMIBC [10]. Although there are no available results yet focusing on the efficacy of immune checkpoint inhibitors (CPIs) in patients with MIBC, many trials in terms of immunotherapy in the neoadjuvant or adjuvant therapy for BLCA are currently recruiting and ongoing [10-13]. Also, encouraged by the promising results of several pivotal trials, various large trials with regard to the approval of the CPIs (avelumab, nivolumab, pembrolizumab, and 
atezolizumab) have been set up to explore the safety and efficacy of them in the treatment for patients of metastatic BLCA [14-17]. Balar et al.'s study demonstrated the efficacy and safety of first-line atezolizumab utilization for cisplatin ineligible patients with metastatic and locally advanced urothelial cancer, as well as the efficacy and safety of pembrolizumab for these patients $[18,19]$. Nevertheless, there has been no signature based on immunerelated genes with prognostic ability which can assess the individual immune status.

In this study, transcriptome data of BLCA patients were downloaded from The Cancer Genome Atlas (TCGA). We constructed and validated an immune signature which consists of 10 immune-related genes. Then, we evaluated the association between this signature and patients' survival outcome and clinical features. In addition, a network of tumorrelated transcription factors (TFs) and immune-related genes was developed to further figure out the potential mechanisms of this signature. Finally, we explore the association between the signature and immune cells' infiltration.

\section{Materials and Methods}

2.1. BLCA Patient Information and Immune-Related Genes Predicting Prognosis Risk. The transcriptomic data and matching clinical information of BLCA patients were downloaded from The Cancer Genome Atlas (TCGA) data portal (up to September 18, 2019; https://portal.gdc.cancer.gov). Clinical information of BLCA patients were also downloaded, including age, gender, grade, stage, and TNM classification. The comprehensive list of a total of 2498 immunerelated genes was obtained from the Immunology Database and Analysis Portal (ImmPort) database (https://immport .niaid.nih.gov) [20]. The Wilcoxon test was used to identify the differential gene between tumor and normal tissues. The genes with $\mid \log$ fold change $(\operatorname{LogFC}) \mid>1$ were thought to be differential gene. "Survival" R package and Cox analysis were used to find the differential immune-related gene with prognostic ability $(p<0.01)$.

2.2. Network between Immune-Related Genes and Transcription Factors. The list of a total of 318 TFs was attained from the Cistrome database (http://cistrome.org/ CistromeCancer/). Correlation analysis between differential immune-related genes predicting prognosis and TFs was done using cor.test. Cytoscape 3.6.1 was used for network graph drawing.

\subsection{Immune-Related Risk Signature Construction and} Performance Assessment. The Cox proportional hazards model was performed to identify the best immune-related gene model for predicting the prognosis in BLCA patients [21]. All patients were divided into high- and low-risk groups according to median immune-related risk score based on the model. The Kaplan-Meier (K-M) survival curve was performed to exhibit the overall survival (OS) of the two groups. ROC curve was performed and the area under the curve (AUC) was calculated to evaluate the prognostic capability of the immune-related risk signature [22]. The univariate and multivariate analyses of survival were put up for both clinicopathologic features and immune signature. Correlation analysis between genes involved in the signature and clinicopathologic features was also performed.

2.4. Correlation Analysis of the Signature and Immune Cells. The concentration of six types of immune cells in all samples in TCGA database, including neutrophil, macrophage, dendritic cell, B cell, CD4 T cell, and CD8 T cell, was downloaded from the Tumor Immune Estimation Resource (TIMER) database (http://cistrome.dfci.harvard.edu/TIMER/). Correlation analysis between six types of immune cells and risk score of BLCA patients were then conducted.

2.5. Statistical Analysis. Student's $t$ test was conducted to make statistical comparison. Heatmaps were generated using "Pheatmap" R package. The Kaplan-Meier (K-M) survival curves were generated utilizing the "survival" $\mathrm{R}$ package. "SurvivalROC" R package was used to produce ROC curve. All of our analysis was conducted using the $\mathrm{R}$ software version 3.5.0 (https://www.r-project.org/). A $p$ value $<0.05$ was thought to be statistically significant.

\section{Results}

3.1. Identification of Prognostic Immune-Related Genes and Transcription Factors and Network Development. To make our study clearer, a workflow of it is shown in Figure 1. A total of 433 BLCA patients' RNA-seq data were collected from TCGA database. The patients' clinical and survival information were summarized in Table 1. A total of 4876 differential genes between tumor and normal samples, with 1423 downexpression genes and 3453 upexpression genes, were identified. The heatmap and volcano figure are shown in Figure 2(a). Among these differential genes, 120 upexpression and 140 downexpression immune-related genes were recognized (Figure 2(b)). After univariate analysis $(p<0.01)$, 24 immune-related genes with prognostic ability were identified, and the forest plot is shown in Figure 2(c). As shown in Figure 2(d), 41 upexpression and 36 downexpression TF genes were recognized from the differential genes. Then, correction analysis was down for the 24 immune-related genes and differential TF genes using cor.test (corFilter $=0.4$ ). The interactions between TFs and immune-related genes are graphically demonstrated in Figure 2(e).

3.2. Construction and Validation of the Immune-Related Risk Signature. To construct an immune-related risk signature, we used "survival" R package to build proportional hazards model. Eventually, 10 genes were elected from the 24 immune-related genes to form an immune-related risk signature, including 8 relatively high-expression genes (MMP9, RBP7, PDGFRA, AHNAK, OLR1, RAC3, IGF1, and AGTR1) and 2 relatively low-expression genes (OAS1, SLIT2). The multivariate analysis of the 10 genes is shown in Table 2, and the K-M analysis of each gene is exhibited in Figure 3. To validate our signature, the risk score of patients was evaluated according to the coefficient value of the 10 genes as follows: risk score $=(0.0003617 * \mathrm{MMP} 9)+(0.0096326 *$ RBP7 $)+(0.0399101 *$ PDGFRA $)+(0.014035 *$ AHNAK $)+$ 


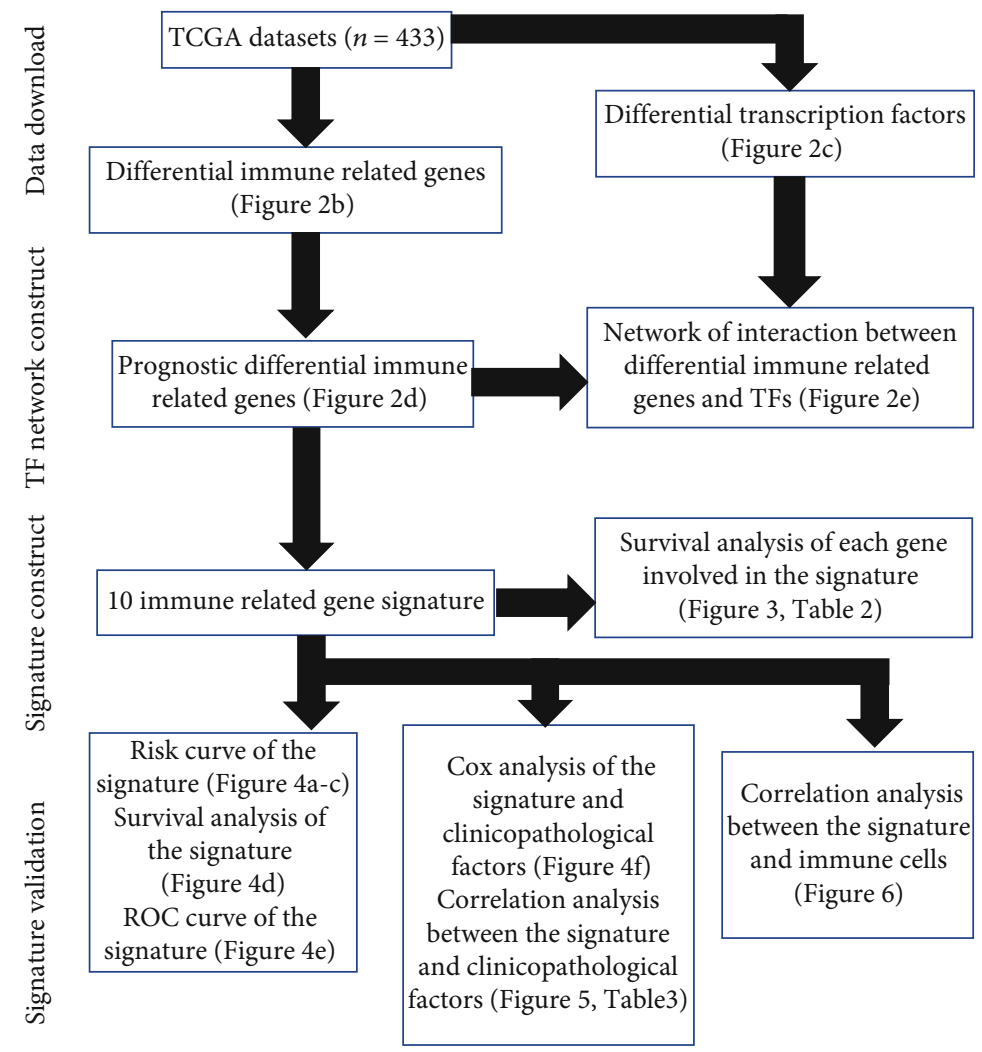

FIGURE 1: The workflow demonstrating the schematic overview of the project.

TABLE 1: Summary of clinical and pathological features of BLCA patients.

\begin{tabular}{|c|c|c|c|}
\hline Variable & $\begin{array}{l}\text { Number of patients } \\
\quad(n=412)\end{array}$ & Variable & $\begin{array}{l}\text { Number of patients } \\
\quad(n=412)\end{array}$ \\
\hline \multicolumn{2}{|r|}{ Age } & \multicolumn{2}{|r|}{ Gender } \\
\hline$\leq 65$ & 162 & Male & 304 \\
\hline$>65$ & 250 & Female & 108 \\
\hline \multicolumn{2}{|r|}{ State } & \multicolumn{2}{|r|}{ Grade } \\
\hline Alive & 253 & High & 388 \\
\hline \multirow[t]{2}{*}{ Dead } & 159 & Low & 21 \\
\hline & & Unknown & 3 \\
\hline \multicolumn{2}{|r|}{ Stage } & \multicolumn{2}{|r|}{ T stage } \\
\hline Stage I & 2 & $\mathrm{~T} 1$ & 3 \\
\hline Stage II & 131 & $\mathrm{~T} 2$ & 121 \\
\hline Stage III & 141 & T3 & 196 \\
\hline Stage IV & 136 & $\mathrm{~T} 4$ & 59 \\
\hline Unknown & 2 & Unknown & 33 \\
\hline \multicolumn{2}{|r|}{$N$ stage } & \multicolumn{2}{|r|}{$M$ stage } \\
\hline N0 & 239 & M0 & 196 \\
\hline N1 & 167 & M1 & 11 \\
\hline Unknown & 6 & Unknown & 205 \\
\hline
\end{tabular}

$(-0.008116 *$ OAS 1$)+(0.0072663 *$ OLR 1$)+(0.0297334$

$*$ RAC3 $)+(-0.209438 *$ SLIT2 $)+(0.2684786 *$ IGF1 $)+$

$(0.1416244 *$ AGTR1).
Then, the patients were divided into the high-risk group or the low-risk group according to median immune-related risk score, as demonstrated in Figures 4(a)-4(c). The K-M analysis was done and results showed that patients with high risk had a poor overall survival (OS) compared with those with low risk (Figure $4(\mathrm{~d}), p<0.001$ ). After that, the ROC curve analysis of the signature showed the promising predictive value of it for BLCA patients' survival ( $\mathrm{AUC}=0.734$, Figure 4(e)). Furthermore, as shown in Figure 4(f), the univariate Cox analysis revealed significant association between the signature and BLCA patients' OS, as well as stage and $\mathrm{T}$ and $\mathrm{N}$ classification. Multivariate Cox analysis further demonstrated that our immune-related signature could serve as an independent predictor of patients' OS (hazard ratio $(\mathrm{HR})=1.303,95 \%$ confidence intervals $(95 \%$ CI) 1.145 to $1.483, p<0.001)$. The results showed that the signature could be an independent predictor for patients' OS, which indicated that our signature has a strong prognostic ability.

3.3. Correlation of the Immune-Related Risk Signature with Clinicopathologic Features. To further validate the clinical value of the signature in BLCA patients, we evaluated the relationship between the 10-gene immune signature and clinicopathologic features. Patients with high risk tend to be male and have advanced grade, stage, and $\mathrm{T}$ classification (Figure 5). Eight genes except OLR1 and AGTR1 were significantly associated with patients' grade. Six genes except 


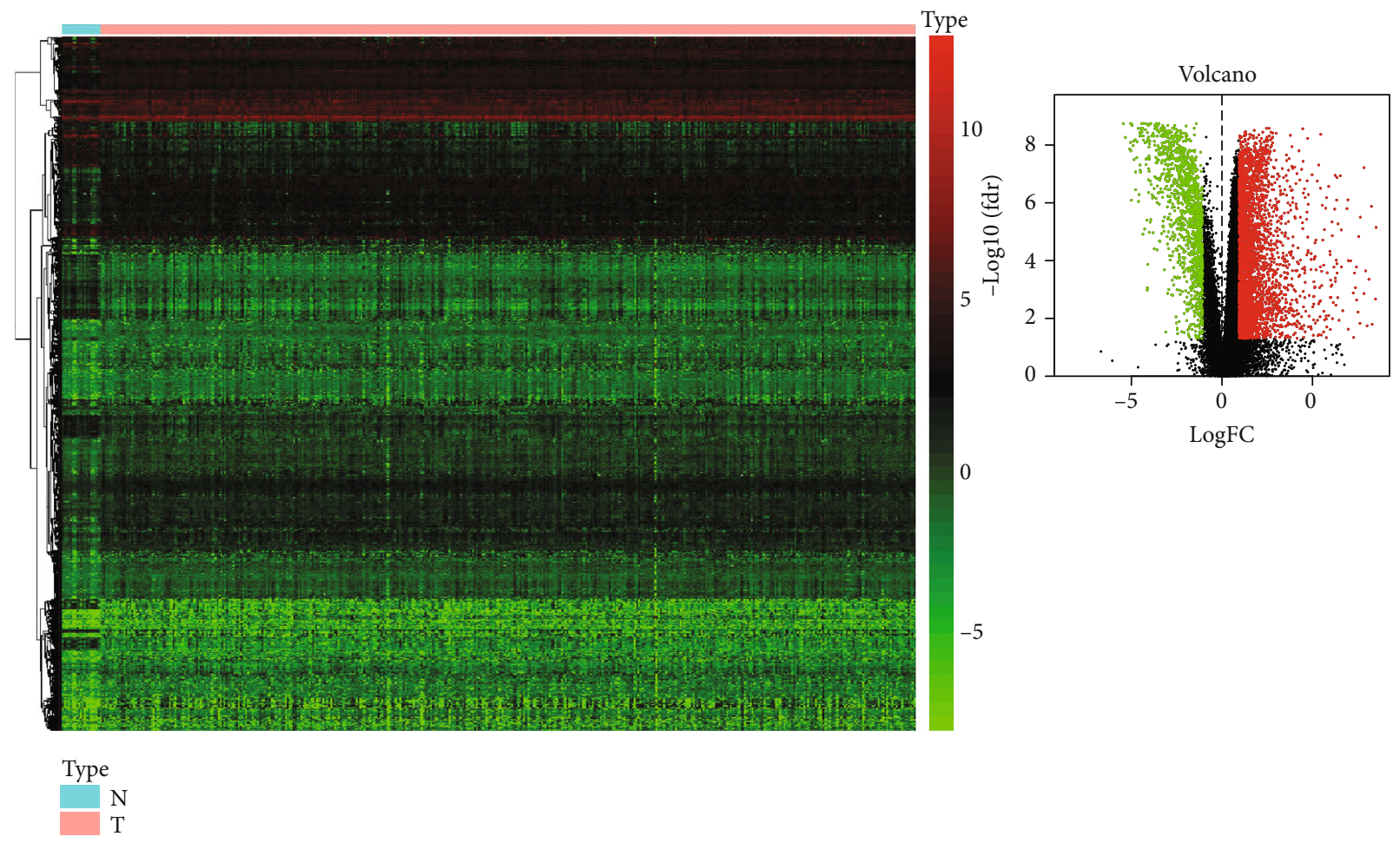

(a)

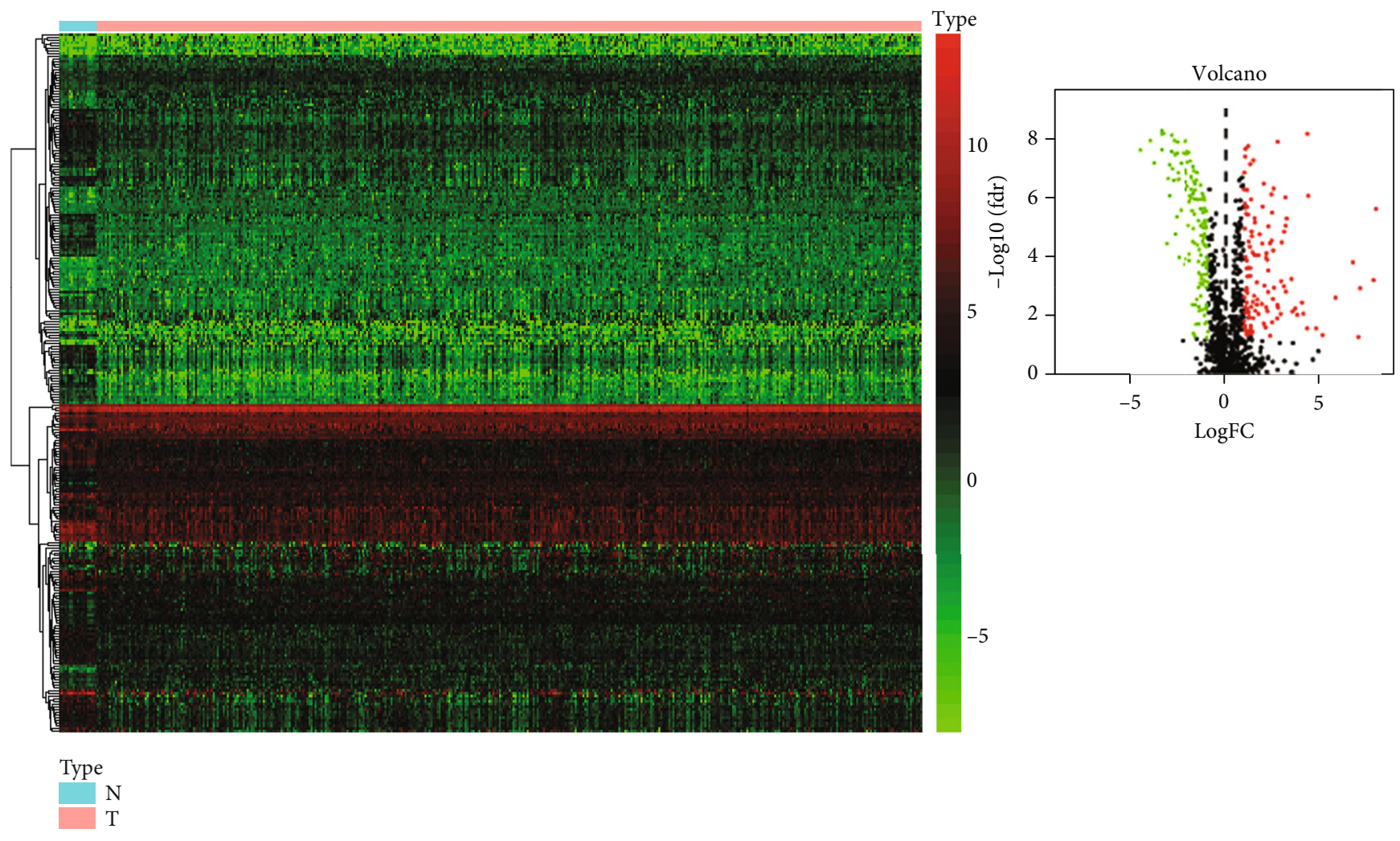

(b)

FIgURE 2: Continued 


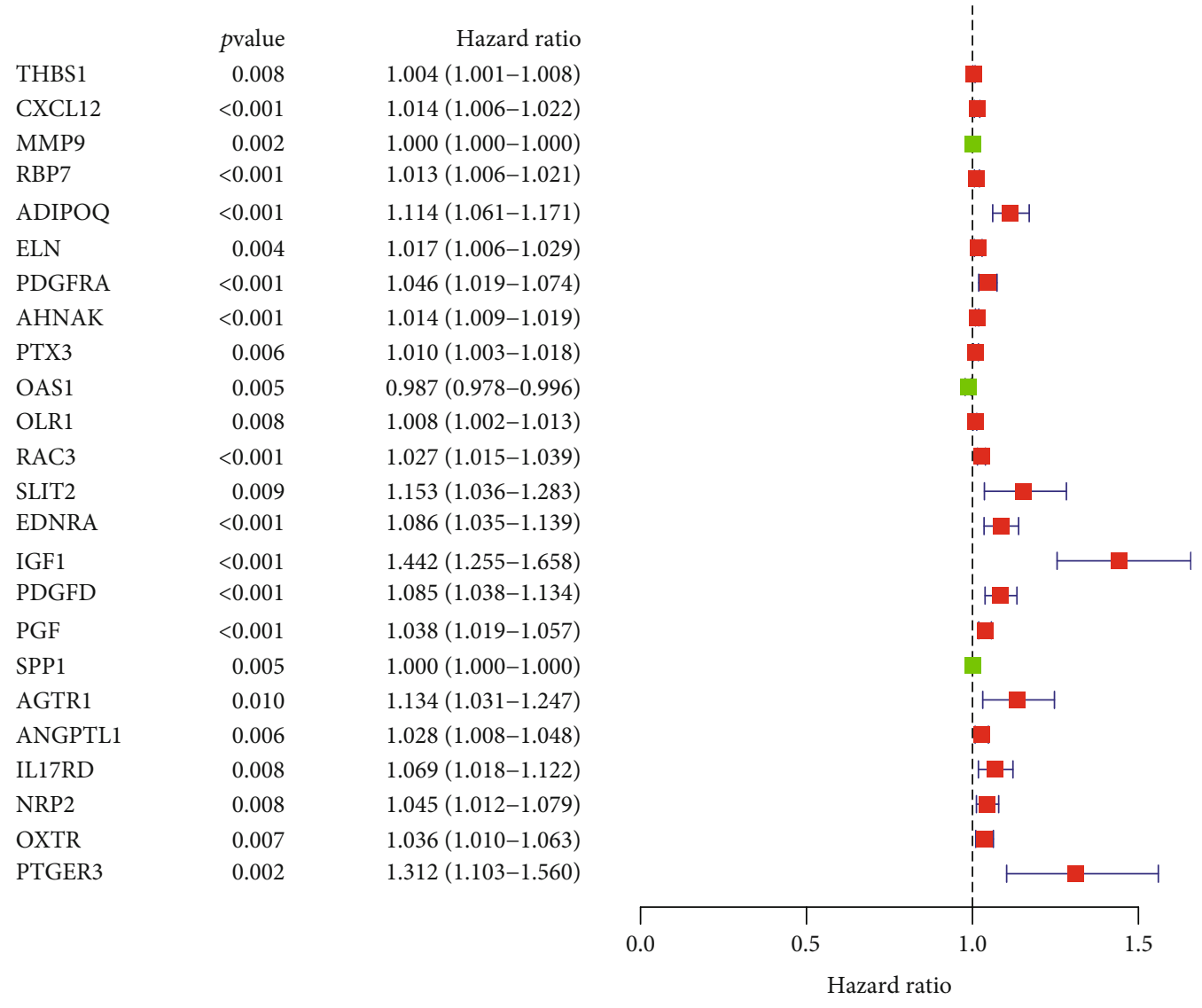

(c)
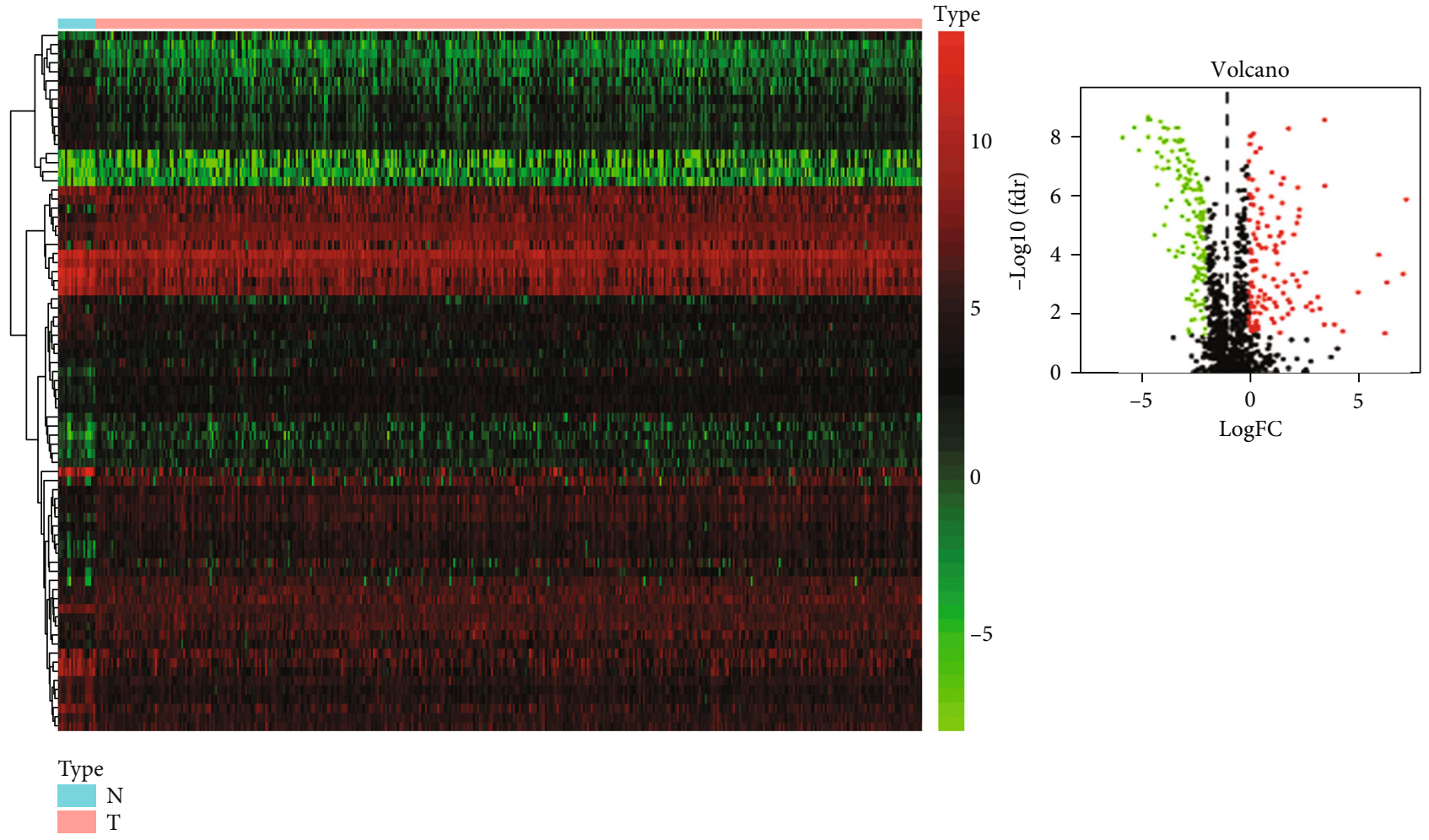

(d)

Figure 2: Continued. 


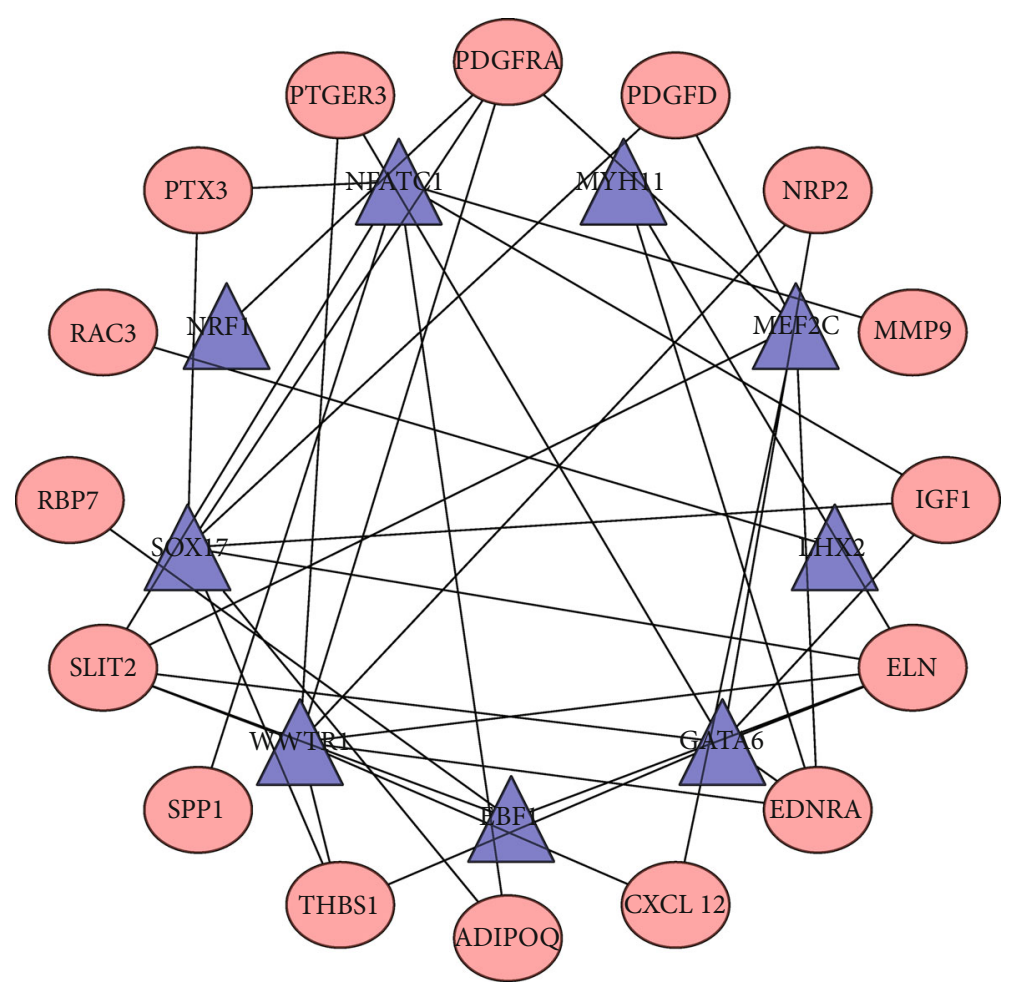

(e)

Figure 2: Heatmap and volcano of (a) differential genes, (b) differential immune-related genes, and (c) differential TFs between tumor and normal samples. Forest plots of hazard ratios of 24 immune-related genes with prognostic ability (d). Network of interaction between differential immune-related genes and TFs (e).

TABLE 2: Multivariate Cox analysis for overall survival of 10 immune-related genes involved in the signature.

\begin{tabular}{lccccc}
\hline id & coef & HR & HR.95L & HR.95H & $p$ value \\
\hline S100B & 0.030657 & 1.031132 & 0.999272 & 1.064006 & 0.055555 \\
MMP9 & 0.000257 & 1.000257 & 1.000065 & 1.00045 & 0.008723 \\
VAV2 & 0.03279 & 1.033334 & 1.010431 & 1.056756 & 0.004138 \\
TYMP & 0.006076 & 1.006095 & 1.003407 & 1.008789 & $8.48 E-06$ \\
ARTN & 0.043398 & 1.044353 & 0.992476 & 1.098942 & 0.095033 \\
BDNF & 0.567795 & 1.764373 & 1.191978 & 2.611635 & 0.004545 \\
DKK1 & 0.002473 & 1.002476 & 0.999753 & 1.005207 & 0.074803 \\
AVPR2 & 0.629915 & 1.877451 & 1.320914 & 2.668471 & 0.000446
\end{tabular}

CI: confidence interval; HR: hazard ratio.

MMP9, OLR1, RAC3, and ATTR1 were significantly associated with patients' stage. The other association of the genes and clinicopathologic factors is also demonstrated in Table 3.

3.4. Association between the Immune-Related Risk Signature and Immune Cells. In order to further explore immunerelated potential mechanisms of the signature, we appraised the association of our signature and six types of immune cells, including neutrophil, macrophage, dendritic cell, B cell,
CD4 T cell, and CD8 T cell. We found that high-risk group patients tended to have more macrophage cell infiltration and no significant change in other five kinds of immune cells $(p<0.05$, Figure 6).

\section{Discussion}

It is believed that BLCA is a complex and intractable disease with high morbidity and mortality which necessitates longterm monitoring [23]. Therefore, both diagnosis ability and treatment of BLCA patients are urgently needed to be improved. With the dramatic development of immunooncology and tumor microenvironment in recent years, immune environment is conformed to play more and more important role in the development of cancer [24]. Therefore, it is essential to explore an immune-related signature which can not only provide immune-related biomarkers for BLCA patients' prognosis but also may serve as a momentous reference in immunotherapy.

In this study, we constructed a stable immune-related signature consisted of 10 immune genes with prognostic ability for BLCA patients utilizing TCGA BLCA dataset. The signature may represent the status of BLCA patients' TIM and prognosis and provide potential targets for immunotherapy. We then evaluated the correlation of the signature and patients' OS and clinicopathological factors to validate its clinical values. The results showed that patients with high 

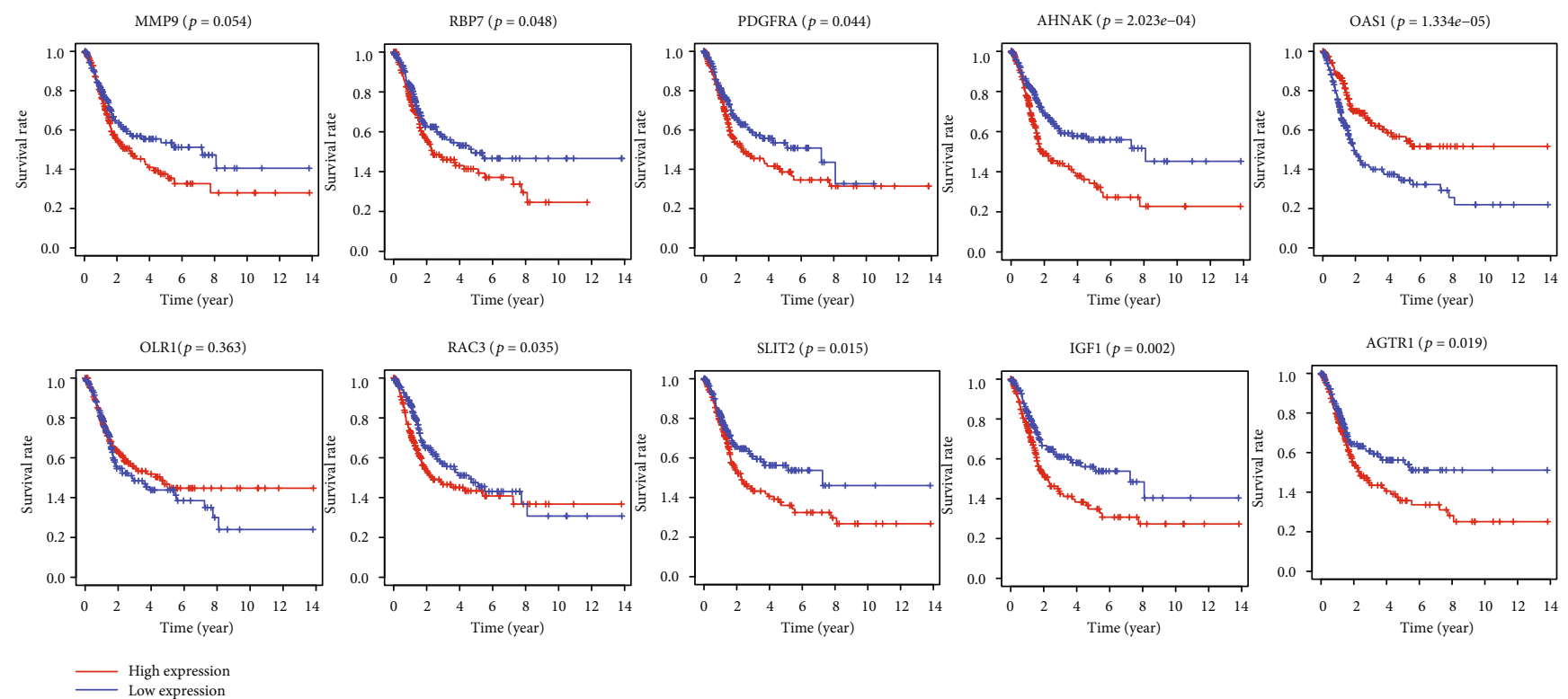

FIgURE 3: The K-M analysis of the 10 immune-related genes used to construct the immune-related risk signature for BLCA, including RBP7, PDGFRA, AHNAK, RAC3, IGF1, AGTR1, SLIT2, OAS1, MMP9, and OLR1.

immune risk have a poor OS and tend to be male and have advanced grade, staging, and T stage. These suggest that the TIM of patients with high immune risk can promote the development and therefore lead to advanced stage and grade. In addition, multivariate analysis further confirms that the signature can be an independent predictor for BLCA patients. Therefore, the immune-related signature can not only predict BLCA patients' survival outcome but also indicate the disease progression.

In order to make out the molecular mechanisms of this immune-related signature, we investigated the genes involved in the signature. Interestingly, IGF1 was the most significant immune gene in the univariate analysis of the 10 immune-related genes' signature $(\mathrm{HR}=1.3080, p=$ $0.0016)$. Insulin-like growth factors (IGFs) are known regulator of energy metabolism and growth. IGF1 belongs to the IGF family and is a part of the metabolic system that includes insulin and two adipocytokines (leptin and adiponectin) [25-28]. More studies reported the role of IGF1 in the development of BLCA. Dunn et al. reported that reduced serum IGF1 would suppress bladder tumor progression in p53-deficient mice [29]. IGF1 can block apoptosis in human bladder cancer cells and increase circulating IGF1, thereby augmenting risk of BLCA patients [30, 31]. Long et al. reported that increased IGF1 can promote cisplatin resistance in bladder cancer cells. Therefore, we suggest that IGF1 may bridge metabolic system and immune oncology. In the signature, SLIT2 is another gene attracting our attention. Sherchan et al. reported that recombinant SLIT2 attenuates neuroinflammation by inhibiting peripheral immune cell infiltration [32]. Similar results were also found in other studies. Chaturvedi et al. reported that SLIT2 can prevent neutrophil recruitment and Guan et al.'s study showed that SLIT2 can inhibit the development of immune responses [33, 34]. More interestingly, the interaction network showed that upstream transcription factor GATA6 and NFATC1 can both bind to IGF1 and SLIT2 which indicated the potential importance of TGF1 and SLIT2 in immune responses. Furthermore, macrophages tend to infiltrate in high-risk group patients. All these results reflect the importance of our immune-related signature in the BLCA microenvironment.

Taken together, based on our knowledge, this is the first study that identified the 10 immune-related genes' signature which can not only be an independent predictor for BLCA patients' survival outcome but also may provide novel targets for immunotherapy for them. Another advantage of this study was using massive data from TCGA database to build and validate the immune-related signature. Nonetheless, several limitations still exist. Firstly, this is a retrospective study with relatively limited samples. Then, we did not validate the signature with external data although we have verified the validity and stability of the signature in various aspects. Finally, the signature should be tested for its predictive ability in the clinical environment and the 10 immune-related genes also need to be further explored.

\section{Conclusions}

In conclusion, the present study constructed an immunerelated signature containing a total of 10 immune-related genes, including MMP9, RBP7, PDGFRA, AHNAK, OAS1, OLR1, RAC3, SLIT2, IGF1, and AGTR1. This signature expressed a strong prognostic ability and served as an independent predictor for BLCA patients' survival and was significantly associated with gender, stage, grade, and $\mathrm{T}$ 


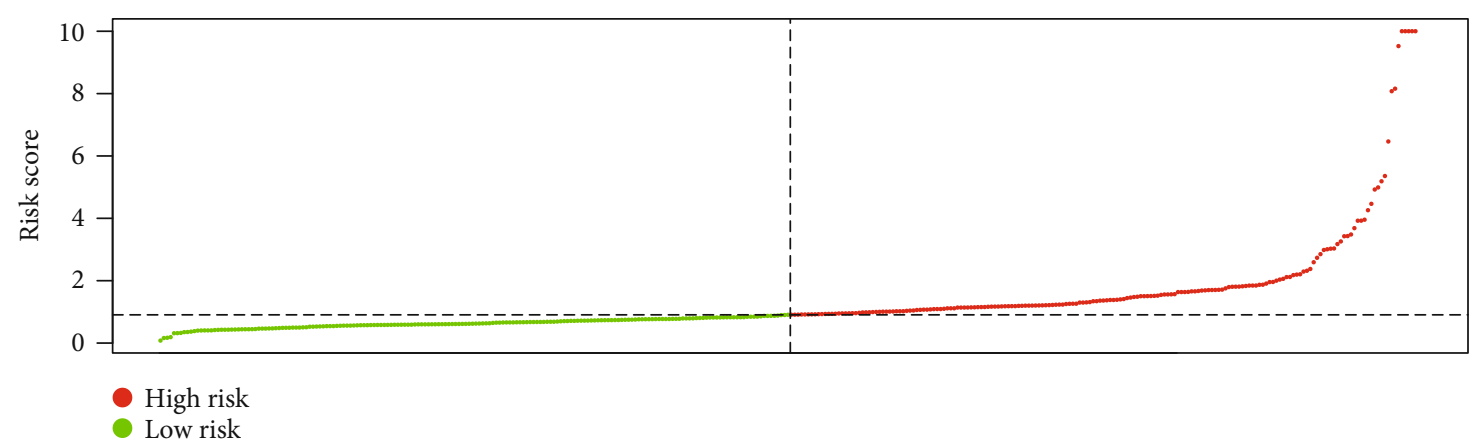

(a)

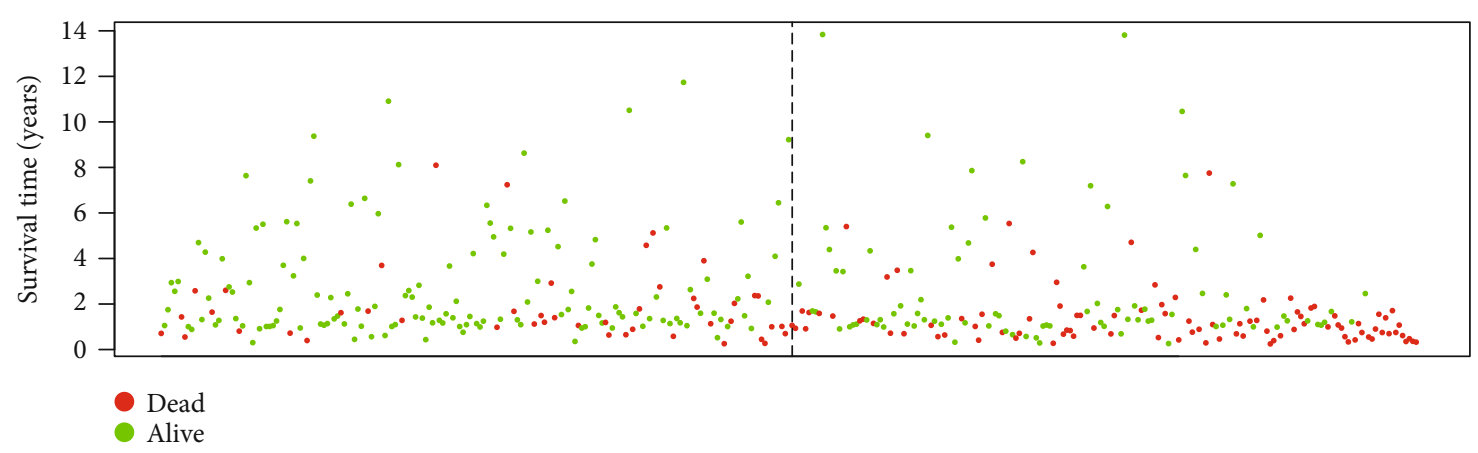

(b)

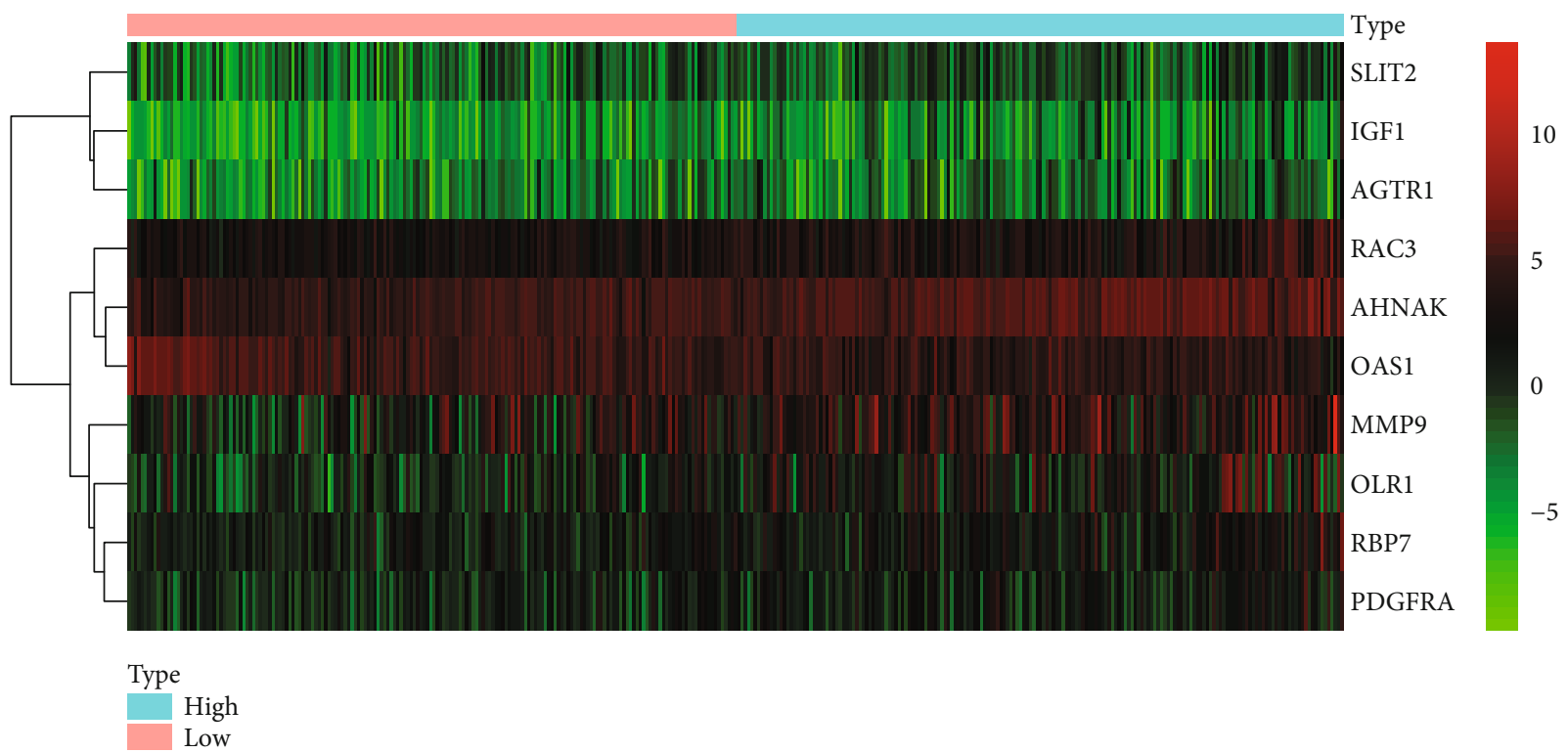

(c)

Figure 4: Continued. 


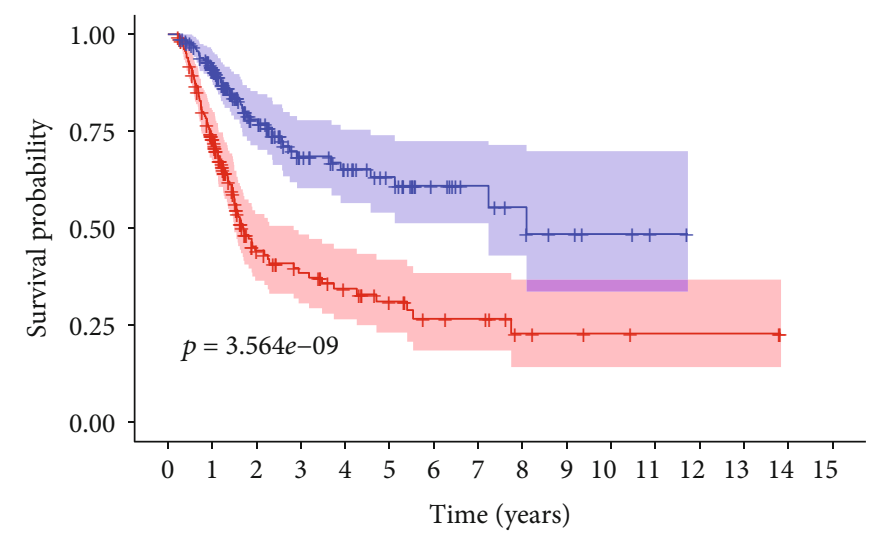

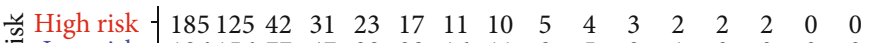
幽 Low risk $\begin{array}{rrrrrrrrrrrrrrrrr}186 & 156 & 77 & 47 & 38 & 28 & 16 & 11 & 8 & 5 & 3 & 1 & 0 & 0 & 0 & 0 \\ 0 & 1 & 2 & 3 & 4 & 5 & 6 & 7 & 8 & 9 & 10 & 11 & 12 & 13 & 14 & 15\end{array}$ Time (years)

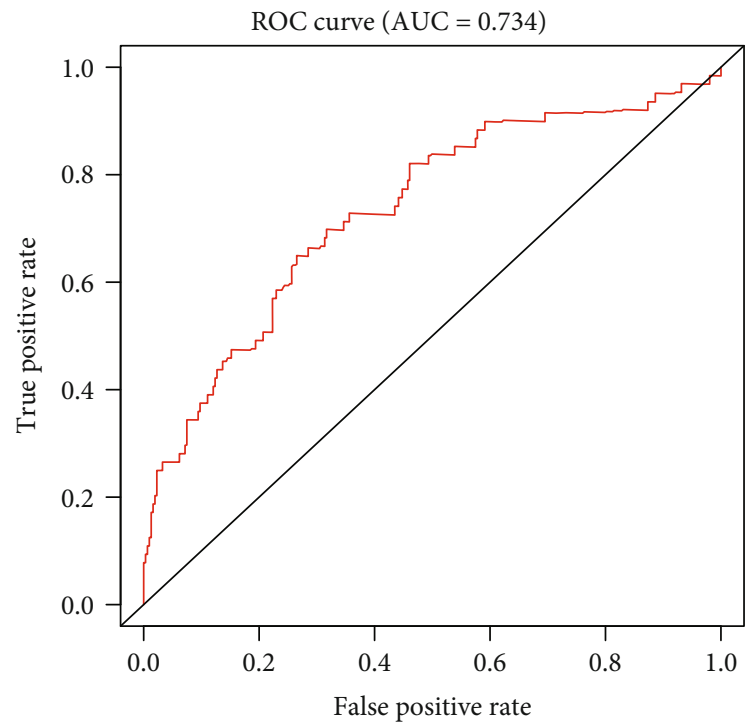

High risk

Low risk

(d)

$\begin{array}{lrr} & \text { pvalue } & \text { Hazard ratio } \\ \text { Age } & 0.080 & 1.027(0.997-1.058) \\ \text { Gender } & 0.096 & 0.604(0.333-1.094) \\ \text { Stage } & 0.001 & 1.913(1.292-2.833) \\ \mathrm{T} & 0.011 & 1.721(1.130-2.621) \\ \mathrm{M} & 0.280 & 1.909(0.590-6.176) \\ \mathrm{N} & 0.008 & 1.501(1.114-2.023) \\ \text { RiskScore } & <0.001 & 1.353(1.222-1.499)\end{array}$

(e)

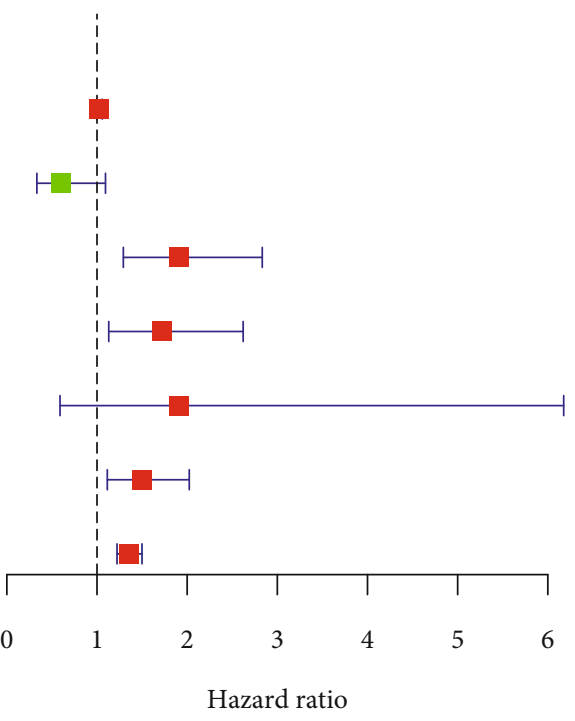

(f)

Figure 4: Continued. 


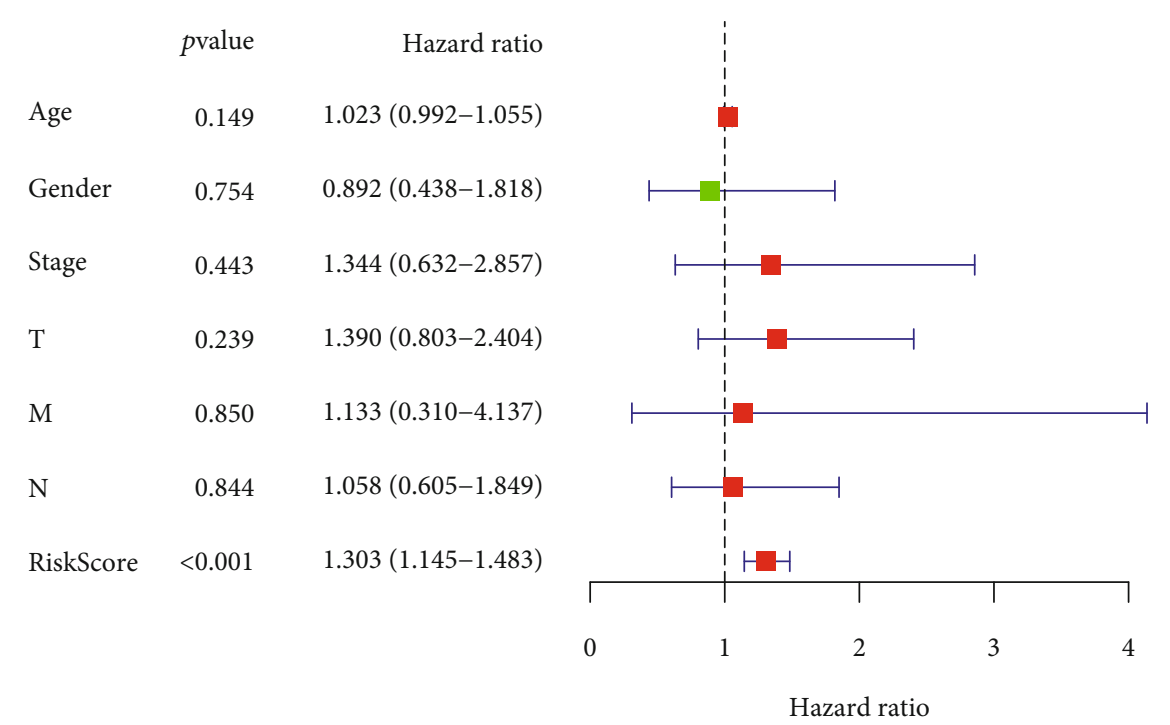

$(\mathrm{g})$

FIGURE 4: Heatmap of the signature consisting of 10 immune-related genes used to construct the immune-related risk signature for BLCA (a-c). The Kaplan-Meier survival analysis of the signature (d). ROC curve analysis of the signature (e). The Cox analysis of the signature and clinicopathological factors: the univariate Cox analysis (f); the multivariate Cox analysis (g).

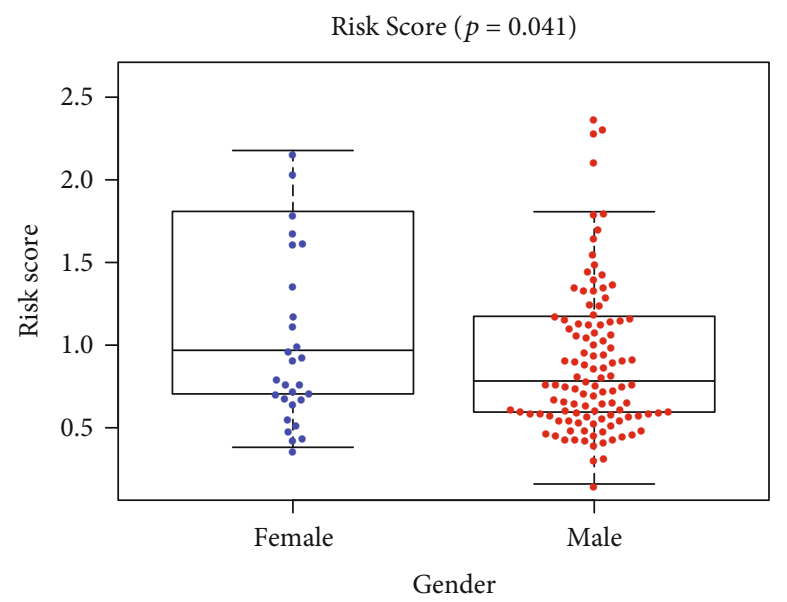

(a)

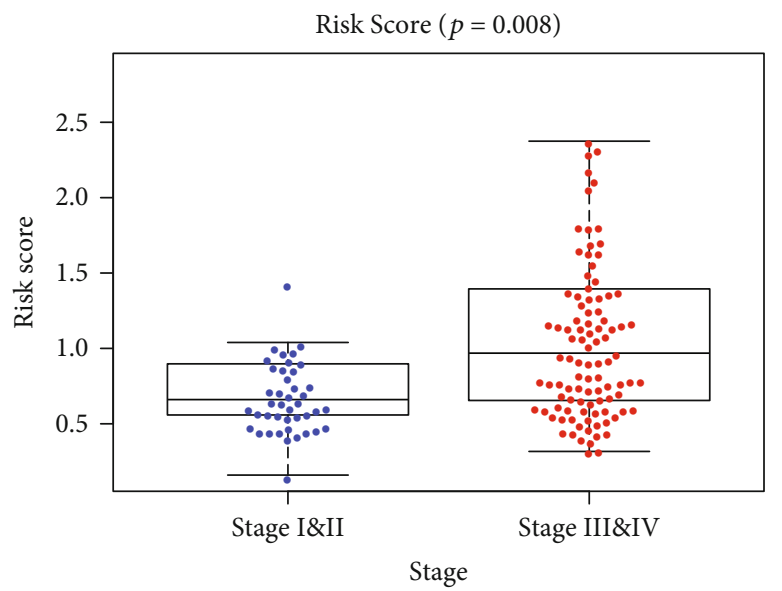

(c)

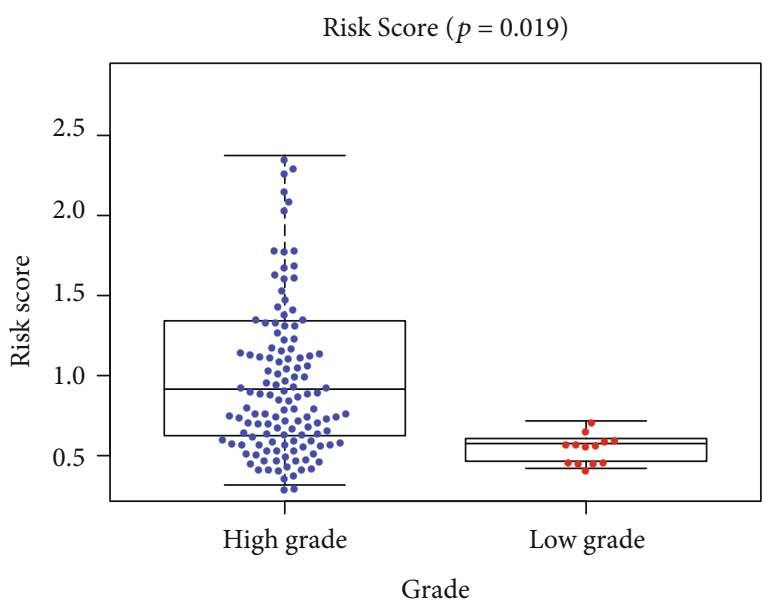

(b)

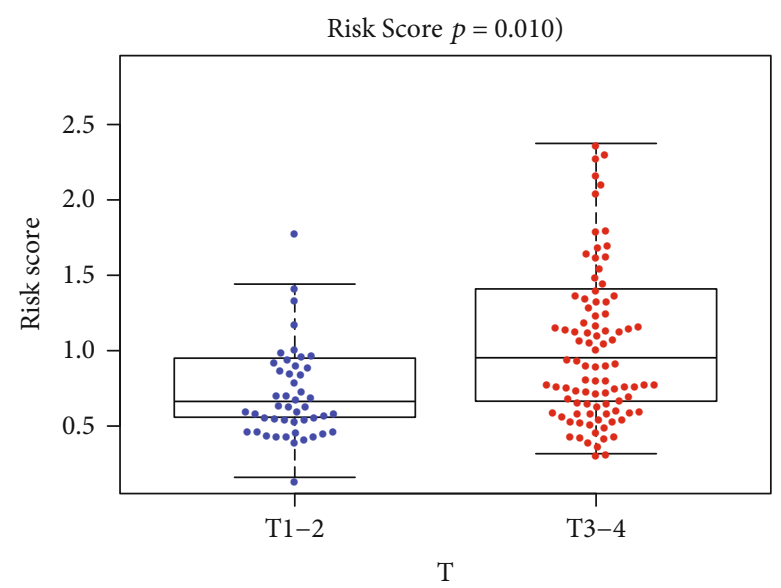

(d)

FIgURE 5: The scatter plot of the relationship between the signature and (a) gender, (b) grade, (c) staging, and (d) T stage. 
TABLE 3: The relationship between the signature and clinical features.

\begin{tabular}{|c|c|c|c|c|c|c|c|}
\hline id & Age & Gender & Grade & Stage & $\mathrm{T}$ & $\mathrm{M}$ & $\mathrm{N}$ \\
\hline MMP9 & $0.005(0.996)$ & $0.569(0.571)$ & $5.384(2.875 e-07)$ & $-1.611(0.110)$ & $-1.966(0.051)$ & $-1.098(0.319)$ & $-0.592(0.555)$ \\
\hline RBP7 & $-0.7(0.485)$ & $1.125(0.268)$ & $2.232(0.027)$ & $-2.055(0.042)$ & $-1.859(0.066)$ & $-1.316(0.245)$ & $-1.611(0.114)$ \\
\hline PDGFRA & $-0.422(0.674)$ & $0.778(0.442)$ & $3.048(0.004)$ & $-3.046(0.003)$ & $-3.008(0.003)$ & $-0.481(0.647)$ & $-1.242(0.218)$ \\
\hline AHNAK & $-0.899(0.370)$ & $1.378(0.176)$ & $5.605(7.957 e-07)$ & $-3.667(3.488 e-04)$ & $-3.898(1.461 e-04)$ & $0.926(0.389)$ & $-2.267(0.026)$ \\
\hline OAS1 & $2.082(0.039)$ & $-2.634(0.010)$ & $-2.181(0.045)$ & $2.19(0.032)$ & & & \\
\hline OLR1 & $0.702(0.484)$ & $-0.098(0.922)$ & & $-1.059(0.292)$ & $-1.021(0.309)$ & $2.971(0.005)$ & \\
\hline RAC3 & $-0.491(0.624)$ & $0.868(0.390)$ & $2.724(0.009)$ & $-0.472(0.638)$ & $0.142(0.887)$ & $-1.139(0.306)$ & \\
\hline SLIT2 & $-0.968(0.335)$ & $1.006(0.320)$ & & $-3.546(5.295 e-04)$ & $-3.545(5.237 e-04)$ & $-1.724(0.144)$ & \\
\hline IGF1 & $0.152(0.879)$ & $0.776(0.442)$ & $2.909(0.004)$ & $-3.54(5.794 e-04)$ & $-3.087(0.003)$ & $-0.981(0.365)$ & $-1.126(0.264)$ \\
\hline AGTR1 & $-1.482(0.141)$ & $0.54(0.592)$ & $-0.795(0.439)$ & $0.37(0.712)$ & $0.427(0.670)$ & $0.032(0.976)$ & $0.719(0.473)$ \\
\hline riskScore & $-0.297(0.767)$ & $2.126(0.041)$ & $2.479(0.019)$ & $-2.683(0.008)$ & $-2.595(0.010)$ & $-0.931(0.394)$ & $-1.806(0.075)$ \\
\hline
\end{tabular}
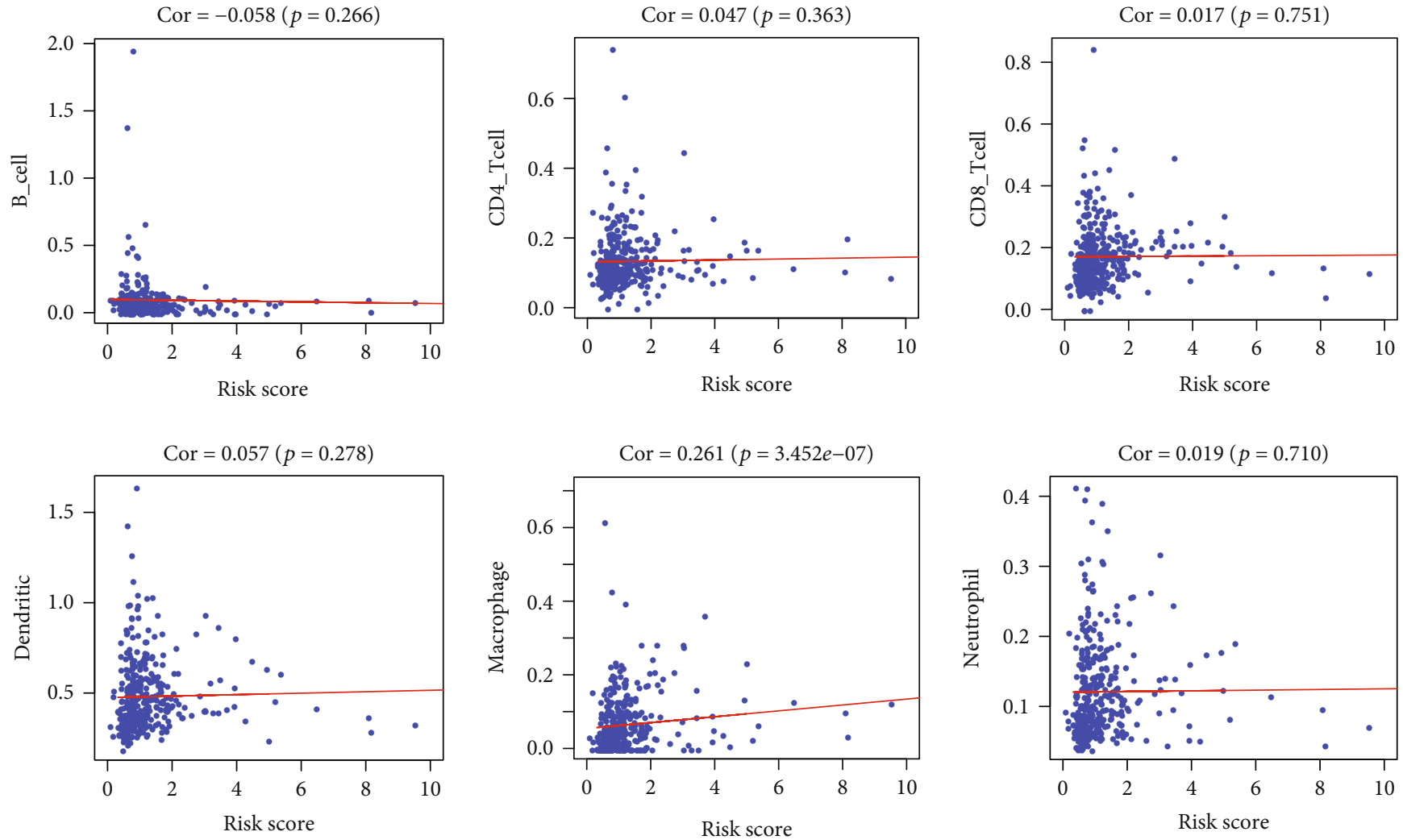

FIGURE 6: The scatter plot of the relationship between the signature and immune cells.

classification. High-risk score group patients presented more macrophage cell infiltration. The findings of this study provide potential novel targets and may promote individualized immunotherapy.

\section{Data Availability}

The data that supported the findings of this study were derived from The Cancer Genome Atlas (TCGA) data portal (https://portal.gdc.cancer.gov), the Immunology Database and Analysis Portal (ImmPort) database (https://immport .niaid.nih.gov), and the Cistrome database (http://cistrome .org/CistromeCancer/). It was also available from the corresponding author.

\section{Conflicts of Interest}

The authors confirm that there are no conflicts of interest.

\section{Authors' Contributions}

The study concept and design was done by Yaofei Jiang, Zhenhong Zou, and Bo Liang. The downloading of data 
and analysis were done by Yaofei Jiang; drafting of the manuscript was done by Yaofei Jiang; Yaofei Jiang, Yibing Wang, Cong Li, and Bo Liang were responsible for the revision of the article.

\section{Acknowledgments}

This research was supported by the National Natural Science Foundation of China (No. 81860420 and No. 81760438).

\section{References}

[1] F. Bray, J. Ferlay, I. Soerjomataram, R. L. Siegel, L. A. Torre, and A. Jemal, "Global cancer statistics 2018: GLOBOCAN estimates of incidence and mortality worldwide for 36 cancers in 185 countries," CA: a Cancer Journal for Clinicians, vol. 68, no. 6, pp. 394-424, 2018.

[2] J. Ferlay, I. Soerjomataram, R. Dikshit et al., "Cancer incidence and mortality worldwide: sources, methods and major patterns in GLOBOCAN 2012," International Journal of Cancer, vol. 136, no. 5, pp. E359-E386, 2015.

[3] M. G. K. Cumberbatch, I. Jubber, P. C. Black et al., "Epidemiology of bladder cancer: a systematic review and contemporary update of risk factors in 2018," European Urology, vol. 74, no. 6, pp. 784-795, 2018.

[4] M. Matuszewski, B. Szymańska, A. Długosz, B. Małkiewicz, J. Dembowski, and A. Piwowar, "Preliminary evaluation of the diagnostic usefulness of uroplakin 2 with an assessment of the antioxidant potential of patients with bladder cancer," BioMed Research International, vol. 2018, Article ID 8693297, 9 pages, 2018.

[5] M. Babjuk, W. Oosterlinck, R. Sylvester et al., "EAU guidelines on non-muscle-invasive urothelial carcinoma of the bladder, the 2011 update," European Urology, vol. 59, no. 6, pp. 9971008, 2011.

[6] A. M. Kamat, J. A. Witjes, M. Brausi et al., "Defining and treating the spectrum of intermediate risk nonmuscle invasive bladder cancer," The Journal of Urology, vol. 192, no. 2, pp. 305-315, 2014.

[7] R. J. Sylvester, A. P. M. van der Meijden, W. Oosterlinck et al., "Predicting recurrence and progression in individual patients with stage Ta T1 bladder cancer using EORTC risk tables: a combined analysis of 2596 patients from seven EORTC trials," European Urology, vol. 49, no. 3, pp. 466-477, 2006.

[8] J. P. Stein, G. Lieskovsky, R. Cote et al., "Radical cystectomy in the treatment of invasive bladder cancer: long-term results in 1, 054 patients," Journal of Clinical Oncology, vol. 19, no. 3, pp. 666-675, 2001.

[9] J. A. Joyce and D. T. Fearon, "T cell exclusion, immune privilege, and the tumor microenvironment," Science, vol. 348, no. 6230 , pp. 74-80, 2015.

[10] A. Morales, D. Eidinger, and A. W. Bruce, "Intracavitary Bacillus Calmette-Guerin in the treatment of superficial bladder tumors," The Journal of Urology, vol. 197, no. 2S, pp. S142S145, 2017.

[11] J. A. Witjes, T. Lebret, E. M. Compérat et al., "Updated 2016 EAU Guidelines on Muscle-invasive and Metastatic Bladder Cancer," European Urology, vol. 71, no. 3, pp. 462-475, 2017.

[12] H. B. Grossman, R. B. Natale, C. M. Tangen et al., "Neoadjuvant chemotherapy plus cystectomy compared with cystectomy alone for locally advanced bladder cancer," The New
England Journal of Medicine, vol. 349, no. 9, pp. 859-866, 2003.

[13] V. L. Chiou and M. Burotto, "Pseudoprogression and immune-related response in solid tumors," Journal of Clinical Oncology, vol. 33, no. 31, pp. 3541-3543, 2015.

[14] J. E. Rosenberg, J. Hoffman-Censits, T. Powles et al., “Atezolizumab in patients with locally advanced and metastatic urothelial carcinoma who have progressed following treatment with platinum-based chemotherapy: a single-arm, multicentre, phase 2 trial," Lancet, vol. 387, no. 10031, pp. 1909-1920, 2016.

[15] P. Sharma, M. K. Callahan, P. Bono et al., "Nivolumab monotherapy in recurrent metastatic urothelial carcinoma (CheckMate 032): a multicentre, open-label, two-stage, multi-arm, phase 1/2 trial," The Lancet Oncology, vol. 17, no. 11, pp. 1590-1598, 2016.

[16] P. Sharma, M. Retz, A. Siefker-Radtke et al., "Nivolumab in metastatic urothelial carcinoma after platinum therapy (CheckMate 275): a multicentre, single-arm, phase 2 trial," The Lancet Oncology, vol. 18, no. 3, pp. 312-322, 2017.

[17] J. Bellmunt, R. de Wit, D. J. Vaughn et al., "Pembrolizumab as second-line therapy for advanced urothelial carcinoma," The New England Journal of Medicine, vol. 376, no. 11, pp. 10151026, 2017.

[18] A. V. Balar, M. D. Galsky, J. E. Rosenberg et al., “Atezolizumab as first-line treatment in cisplatin-ineligible patients with locally advanced and metastatic urothelial carcinoma: a single-arm, multicentre, phase 2 trial," Lancet, vol. 389, no. 10064, pp. 67-76, 2017.

[19] A. V. Balar, D. E. Castellano, P. H. O'Donnell et al., "Pembrolizumab as first-line therapy in cisplatin-ineligible advanced urothelial cancer: results from the total KEYNOTE-052 study population," Journal of Clinical Oncology, vol. 35, 6_suppl, p. 284, 2017.

[20] S. Bhattacharya, S. Andorf, L. Gomes et al., "ImmPort: disseminating data to the public for the future of immunology," Immunologic Research, vol. 58, no. 2-3, pp. 234-239, 2014.

[21] N. Simon, J. Friedman, T. Hastie, and R. Tibshirani, "Regularization paths for Cox's proportional hazards model via coordinate descent," Journal of Statistical Software, vol. 39, no. 5, pp. 1-13, 2011.

[22] P. J. Heagerty, T. Lumley, and M. S. Pepe, “Time-dependent ROC curves for censored survival data and a diagnostic marker," Biometrics, vol. 56, no. 2, pp. 337-344, 2000.

[23] C. Peng, M. Y. Hua, N. S. Li et al., "A colorimetric immunosensor based on self-linkable dual-nanozyme for ultrasensitive bladder cancer diagnosis and prognosis monitoring," Biosensors \& Bioelectronics, vol. 126, pp. 581-589, 2019.

[24] D. S. Chen and I. Mellman, "Elements of cancer immunity and the cancer-immune set point," Nature, vol. 541, no. 7637, pp. 321-330, 2017.

[25] S. Fischer, M. Hanefeld, S. M. Haffner et al., "Insulin-resistant patients with type 2 diabetes mellitus have higher serum leptin levels independently of body fat mass," Acta Diabetologica, vol. 39, no. 3, pp. 105-110, 2002.

[26] M. Matsubara, S. Maruoka, and S. Katayose, "Inverse relationship between plasma adiponectin and leptin concentrations in normal-weight and obese women," European Journal of Endocrinology, vol. 147, no. 2, pp. 173-180, 2002.

[27] N. Stefan, B. Vozarova, T. Funahashi et al., "Plasma adiponectin concentration is associated with skeletal muscle insulin receptor tyrosine phosphorylation, and low plasma 
concentration precedes a decrease in whole-body insulin sensitivity in humans," Diabetes, vol. 51, no. 6, pp. 18841888, 2002.

[28] M. Wauters, R. V. Considine, J. S. Yudkin, F. Peiffer, I. de Leeuw, and L. F. van Gaal, "Leptin levels in type 2 diabetes: associations with measures of insulin resistance and insulin secretion," Hormone and Metabolic Research, vol. 35, no. 2, pp. 92-96, 2003.

[29] S. E. Dunn, F. W. Kari, J. French et al., "Dietary restriction reduces insulin-like growth factor I levels, which modulates apoptosis, cell proliferation, and tumor progression in p53deficient mice," Cancer Research, vol. 57, no. 21, pp. 4667$4672,1997$.

[30] M. Iwamura, M. Ishibe, P. M. Sluss, and A. T. K. Cockett, "Characterization of insulin-like growth factor I binding sites in human bladder cancer cell lines," Urological Research, vol. 21, no. 1, pp. 27-32, 1993.

[31] H. Zhao, H. B. Grossman, M. R. Spitz, S. P. Lerner, K. Zhang, and $\mathrm{X}$. Wu, "Plasma levels of insulin-like growth factor-1 and binding protein-3, and their association with bladder cancer risk," The Journal of Urology, vol. 169, no. 2, pp. 714-717, 2003.

[32] P. Sherchan, L. Huang, Y. Wang, O. Akyol, J. Tang, and J. H. Zhang, "Recombinant Slit2 attenuates neuroinflammation after surgical brain injury by inhibiting peripheral immune cell infiltration via Robo1-srGAP1 pathway in a rat model," $\mathrm{Neu}$ robiology of Disease, vol. 85, pp. 164-173, 2016.

[33] S. Chaturvedi, D. A. Yuen, A. Bajwa et al., "Slit 2 prevents neutrophil recruitment and renal ischemia-reperfusion injury," Journal of the American Society of Nephrology, vol. 24, no. 8, pp. 1274-1287, 2013.

[34] H. Guan, G. Zu, Y. Xie et al., "Neuronal repellent Slit 2 inhibits dendritic cell migration and the development of immune responses," Journal of Immunology, vol. 171, no. 12, pp. 6519-6526, 2003. 